\title{
Cross-Sectional Age Variance Extraction: What's Change Got To Do With It?
}

\author{
Ulman Lindenberger and Timo von Oertzen \\ Max Planck Institute for Human Development \\ and Saarland University
}

\author{
Paolo Ghisletta \\ University of Geneva, and Distance Learning University, \\ Switzerland
}

\author{
Christopher Hertzog \\ Georgia Institute of Technology
}

\begin{abstract}
In cross-sectional age variance extraction (CAVE), age, the indicator of a hypothesized developmental mechanism, and a developmental outcome are specified as independent, mediator, and target variables, respectively, to test hypotheses about behavioral development. We show that: (a) longitudinal change in a mediator variable accounting for substantial cross-sectional age-related variance in the target variable need not correlate with the target variable's longitudinal change; and, conversely, (b) longitudinal change in a mediator not sharing cross-sectional age-related variance with the target variable may nevertheless correlate highly with that variable's longitudinal change. We discourage use of CAVE for testing multivariate hypotheses about behavioral development.
\end{abstract}

Keywords: cross-sectional data, longitudinal data, variance partitioning, correlated change, longitudinal mediator

A central goal of developmental psychology is to identify the number and nature of causes that drive age-related changes in behavior (Baltes, Reese, \& Nesselroade, 1988; Craik \& Bialystok, 2008; Flavell, 1992; Hertzog, 1985; Kagan, 1980; Rabbitt, 1993; Salthouse, 1994). A critical question, then, is whether age-related changes in one aspect of behavior are brought about by age-related changes in another aspect. For instance, cognitive aging researchers have investigated whether adult age declines in complex cognition are caused by changes in processing speed (e.g., Salthouse, 1996).

Mechanisms of developmental changes are generally not under experimental control (Wohlwill, 1973). Hence, developmental psychologists need to rely on observational research designs and correlational statistical techniques to assess whether developmental change in different variables is correlated (Baltes et al., 1988; Hertzog, 1985, 1996; Lindenberger \& Pötter, 1998). Despite early

Ulman Lindenberger, Max Planck Institute for Human Development; and School of Psychology, Saarland University; Timo von Oertzen, Max Planck Institute for Human Development; and Department of Mathematics, Saarland University; Paolo Ghisletta, Faculty of Psychology and Educational Sciences, University of Geneva, and Distance Learning University, Switzerland; Christopher Hertzog, School of Psychology, Georgia Institute of Technology.

This article was revised while Ulman Lindenberger was a fellow at the Center for Advanced Study in the Behavioral Sciences at Stanford University. The main results of this article were presented in May 2005 at the International Conference on the Future of Cognitive Aging Research, Pennsylvania State University, University Park, PA, and in July 2008 at the XXIX International Congress of Psychology, Berlin, Germany.

Correspondence concerning this article should be addressed to Ulman Lindenberger, Max Planck Institute for Human Development, Lentzeallee 94, 14195 Berlin, Germany. E-mail: lindenberger@mpib-berlin.mpg.de and repeated warnings (e.g., Baltes et al., 1988; Gollob \& Reichardt, 1987; Kalveram, 1965), developmental psychology has relied heavily on cross-sectional samples for this purpose. In line with earlier work (Collins, Graham, \& Flaherty, 1998; Hofer, Flaherty, \& Hoffmann, 2006; Hofer \& Sliwinski, 2001; Lindenberger \& Pötter, 1998; Maxwell \& Cole, 2007; Sliwinski \& Hofer, 1999), this article further critiques the use of cross-sectional data for developmental inference.

The basic principle of cross-sectional age variance extraction (CAVE) can be illustrated with a simple three-variable mediation model (e.g., MacKinnon \& Fairchild, 2009), treating age as the independent variable, $X$ as the mediator variable, and $Y$ as the dependent, or target, variable. CAVE partitions the age-related variance of $Y$ (or the simple effect of age on $Y$ ) into two parts: one part that is statistically predicted by $X$, and the remaining part that is not. The amount of age-related variance in $Y$ predicted by $X$ is termed the shared effect of age (because it is shared with the mediator).

In terms of effect size, the relative importance of the mediator variable as a potential cause for age-related changes in the dependent variable can be expressed as the proportion of total agerelated variance in the dependent variable that is shared with the mediator variable (e.g., Verhaeghen \& Salthouse, 1997; see also Fairchild, MacKinnon, Taborga, \& Taylor, 2009). Following an earlier formal analysis by Lindenberger and Pötter (1998), we term this quantity shared over simple effects (SOS). When the mediator variable predicts all the age-related variance in the dependent variable (i.e., complete mediation), then shared and simple effects are equal, and SOS $=1.0$. Conversely, when the mediator variable does not predict any of the age-related variance in the dependent variable, then $\mathrm{SOS}=0.0$ and mediation is absent (for more information on SOS, see Lindenberger \& Pötter, 1998). 
Lindenberger and Pötter (1998) showed that SOS are a quadratic function of the partial correlation between the mediator variable and the dependent variable controlling for the independent variable. They also noted that for each mediator model there exists a mathematically equivalent model positing that the age effects on $X$ and $Y$ are entirely independent. Hence, Lindenberger and Pötter (1998) concluded that CAVE should not be used when attempting to infer the dimensions and causes of developmental change.

This article adds a temporal dimension to Lindenberger and Pötter's (1998) analysis by acknowledging that age or any other relevant time dimension (e.g., time in study, time after disease onset, or time to death) has a special conceptual and statistical status because it functions as a vector on which observations are aligned (Hofer \& Sliwinski, 2001; Hofer et al., 2006; Wohlwill, 1970). From this perspective, cross-sectional data represent an extreme case of missing data with respect to time. Figure 1 illustrates this point. In its upper panel, the full data set for 75 individuals observed 20 times on variable $Y$ (e.g., reasoning) can be seen. The middle panel highlights the cross-sectional data points (i.e., one for each individual) observed in a hypothetical cross-sectional study. In the lower panel, the change trajectories have been deleted, and all that is left are cross-sectional observations, or one-occasion snapshots. Note that the longitudinal trajectories of variable $Y$ may not resemble those depicted in the upper panel of Figure 1 to reduce to the same set of cross-sectional snapshots. In other words, there is a one-to-many mapping of cross-sectional data to underlying longitudinal trajectories.

Let us now posit an analogous picture for a candidate mediator variable $X$ (e.g., perceptual speed). $X$ would also have been measured once in each individual, at the same point in time as $Y$. On the basis of the covariance matrix among age, $X$, and $Y$, researchers may apply CAVE to test the hypothesis that $X$ mediates age-related variance in $Y$.

Assume it is the case that, indeed, developmental changes in $X$ are driving developmental changes in $Y$. Conceptually, then, it is reasonable to posit that individual differences in rates of change for $X$ and $Y$ ought to be correlated. For instance, individuals who decline more than average in perceptual speed should also decline more than average in reasoning. CAVE presumes that crosssectional data can capture these change relations in some meaningful way. Doubt would be cast on the validity of CAVE for evaluating the structure of behavioral change if it detects statistical mediation in cross-sectional data in the absence of positively correlated changes between $X$ and $Y$ (cf. Maxwell \& Cole, 2007).

This article scrutinizes CAVE from this perspective. First, we express the cross-sectional correlation between $X, Y$, and SOS in terms of a longitudinal latent growth curve model (LGCM; McArdle \& Nesselroade, 2003), using results from Appendixes A and B. Second, by charting SOS as a function of LGCM parameters, we explore the relation between CAVE and correlated changes. Finally, we discuss the implications of our formal analysis and conclude that CAVE is not well suited for testing multivariate hypotheses about developmental change.

\section{Cross-Sectional Age Variance Extraction in Terms of the Latent Growth Curve Model}

To express SOS in terms of bivariate linear change, we parameterize the cross-sectional correlation between two variables in
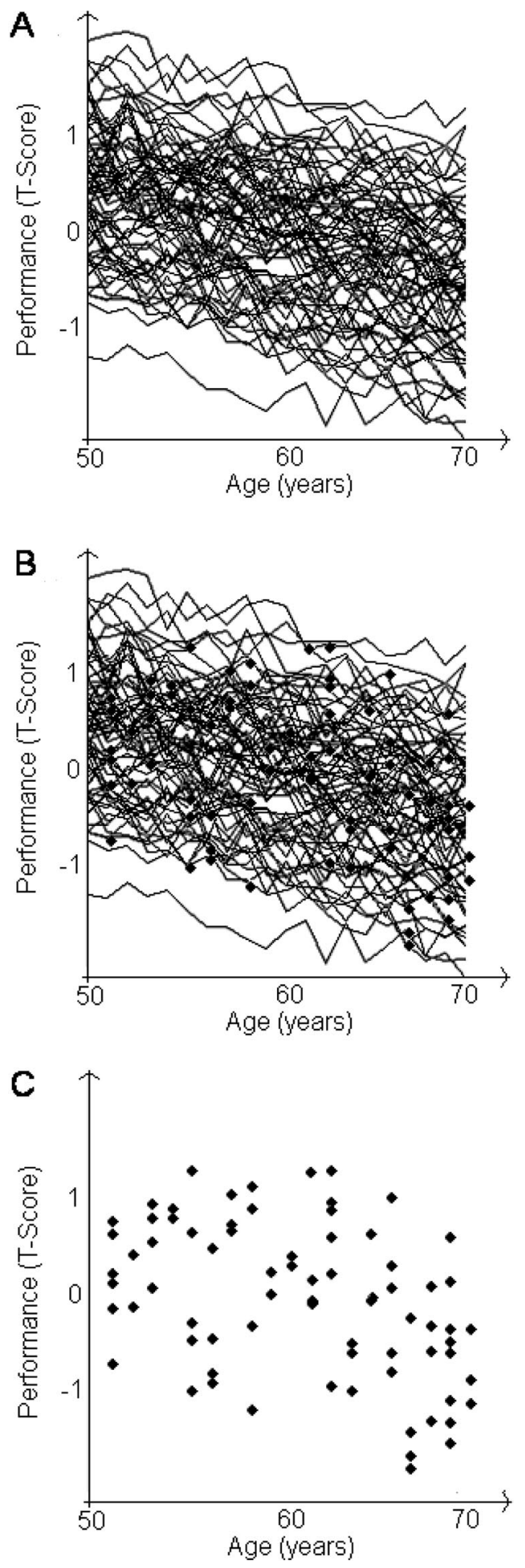

Figure 1. Cross-sectional data as an extreme form of missingness: An illustration. We assume a homogeneous population of individuals with a linear change process, and sample 75 individual longitudinal trajectories from this population (A). Cross-sectional observations are randomly selected for each individual (B) at a given point in time. By deleting the trajectories from which cross-sectional data points were taken $(\mathrm{C})$, we note the noninjective (one-to-many) relation between cross-sectional data and their corresponding longitudinal trajectories. 
terms of a bivariate LGCM. For both variables $X$ and $Y$, individual growth is described by the level at the beginning of the observation period (intercept) and a linear slope capturing a person's change over time $t$ (e.g., chronological age). Each of the four values-two intercepts and two slopes-is drawn from a normal distribution. We also assume normally distributed error terms for $X$ and $Y$ at each occasion, and scale time $t$ from 0 to 1 (the following holds for other time scalings).

In Appendix A, we formally derive the means and variances of $X$ and $Y$, their covariances with time, and the covariance among each other. This allows us to express the cross-sectional correlation between $X$ and $Y$ as a function of LGCM parameters:

$$
\frac{12 \operatorname{covI}_{Y}, I_{X}+6\left(\operatorname{cov} I_{Y}, S_{X}+\operatorname{cov} I_{X}, S_{Y}\right)+4 \operatorname{cov} S_{Y}, S_{X}+\mu S_{Y}, S_{X}}{\sqrt{\left(12 \operatorname{varI}_{Y}+4 \operatorname{var}_{Y}+12 \operatorname{covI} I_{Y}, S_{Y}+\mu_{S_{Y}}^{2}+\theta_{Y}^{2}\right)\left(12 v a r I_{X}+4 v a r S_{X}+12 \operatorname{covI}_{X}, S_{X}+\mu_{S_{X}}^{2}+\theta_{X}^{2}\right)}}
$$

where $I_{X}$ and $I_{Y}$ are the intercepts and $S_{X}$ and $S_{Y}$ the slopes of $X$ and $Y$, respectively, and $\operatorname{varI}_{X}, \operatorname{cov}_{Y}, I_{X}, \mu S_{X}$, and $\theta_{X}$ denote the variance, covariance, mean and error variance of the indexed variable. We note that this equation can be derived from Hofer et al.'s (2006) Equation 2 by distributing expectations over terms and simplifying; see also Appendix A. On the basis of Equation 1, increasing the correlation between the two linear changes $\left(\operatorname{cov} S_{Y}, S_{X}\right)$ increases the cross-sectional correlation between $X$ and $Y$, as expected. Note, however, that several other components of the growth process also contribute to the cross-sectional correlation.

Building on Equation 1, the relation between SOS and LGCM can be derived (see Appendix B). This relation can be summarized as follows: ${ }^{1}$

$$
S O S=1-\frac{12 \operatorname{var} Y\left(\mu S_{Y} \operatorname{var} X-\mu S_{X} \operatorname{cov} Y, X\right)^{2}}{\left(\operatorname{var} Y \operatorname{var} X-\operatorname{cov}_{Y, X}^{2}\right)\left(12 \operatorname{var} X-\mu_{S_{X}}^{2}\right) \mu_{S_{Y}}^{2}}
$$

The expressions $v a r_{X}, v a r_{Y}$, and $\operatorname{cov}_{Y, X}$ for the distribution parameters of $X$ and $Y$ are short notations for LGCM parameters, which are defined in Lemmas 1 and 2 of Appendix A. In what follows, we use Equation 2 to evaluate mediation in CAVE in terms of different constellations of growth curve parameters (i.e., changes in $X$ and $Y$ ).

\section{Illustrating the Properties of CAVE}

The implications of Equation 2 are profound and demonstrate the problematic nature of CAVE for developmental research. To demonstrate this, we first examine the influence of correlated change on SOS for parameter ranges that are typical of longitudinal changes in adult cognition. ${ }^{2}$ Second, we identify a parameter constellation for which higher correlations of longitudinal change in the two variables (i.e., slope-slope correlations) are consistently associated with greater age mediation (i.e., higher SOS) and examine the sensitivity of detecting this association to variation in other parameters.

\section{Example 1: Dependency of SOS Effects on the Slope-Slope Correlation}

Our illustration assumes (without loss of generality) that the two variables are scaled as T scores, with a mean of 50 and a standard deviation of 10 at the first measurement occasion (e.g., age 50). The variance of the two slopes is set to a value of $0.35 S D$ units per year, which corresponds to a variance of 50 for the entire observation interval of 20 years (Hertzog, Lindenberger, Ghisletta, \& von Oertzen, 2006). This amount of variance in change is likely to overestimate rather than underestimate the amount of variance in change, on the basis of what has been empirically observed in longitudinal studies of adult cognitive development (e.g., de Frias, Lövdén, Lindenberger, \& Nilsson, 2007; Hultsch, Hertzog, Dixon, \& Small, 1998; Schaie, 1996). The error variance for both variables is set to 25 , which corresponds to a growth curve reliability of 0.80 at the first occasion (see Hertzog et al., 2006, for further discussion). The cross-sectional correlation between the dependent variable and age is set to $r=-0.25$, which, with the current parameter settings, corresponds to a mean longitudinal decline of $10 \mathrm{~T}$-score units over the entire observation period of 20 years, or the equivalent of 1 standard deviation at the first occasion. The mean longitudinal change of the mediator variable is adapted to express the cross-sectional correlation between the mediator and age, which is varied systematically. To give an impression of the relation between the two parameters in this example, a crosssectional correlation between the mediator and age of $r=-0.65$ corresponds to a mean longitudinal decline of $32 \mathrm{~T}$-score units in 20 years. The correlation between the two variables at baseline (i.e., the intercept-intercept correlation) is fixed to 0.5 , reflecting the positive manifold of intellectual abilities, and the covariances between the two intercepts and the two slopes are both fixed to zero.

The three-dimensional Panel A in Figure 2 represents SOS as a function of the cross-sectional correlation between the mediator variable and age and the correlation between the linear changes (slopes) of the mediator variable and the target variable as described in Equation 2. Note that the influence of the cross-sectional mediator-age correlation on SOS appears stronger than the influence of the slope-slope correlation. Indeed, if we inspect the two-dimensional slice of the diagram at the plane where the slope-slope correlation is zero (see Panel B), CAVE still suggests that the mediator variable explains between $77 \%$ and $100 \%$ of the age-related variance in the dependent variable; the exact value continues to depend on the age correlation of the mediator, despite the fact that the two change processes are uncorrelated. Complete mediation (i.e., $\mathrm{SOS}=1.0$ ) occurs when the age-mediator correlation is about -0.5 , which corresponds to a mean longitudinal decline of $22 \mathrm{~T}$ scores over 20 years, a high but realistic value (see Salthouse, 1996, for similar correlations of age with measures of

\footnotetext{
${ }^{1}$ For brevity, we left the terms for variances of $X$ and $Y$ and the covariance of $X$ and $Y$ in the equation. These three terms can be substituted from the derivations in Appendix A and Equations A7, A8, and A28.

${ }^{2}$ We acknowledge the late Paul B. Baltes, who suggested this line of reasoning.
} 
A

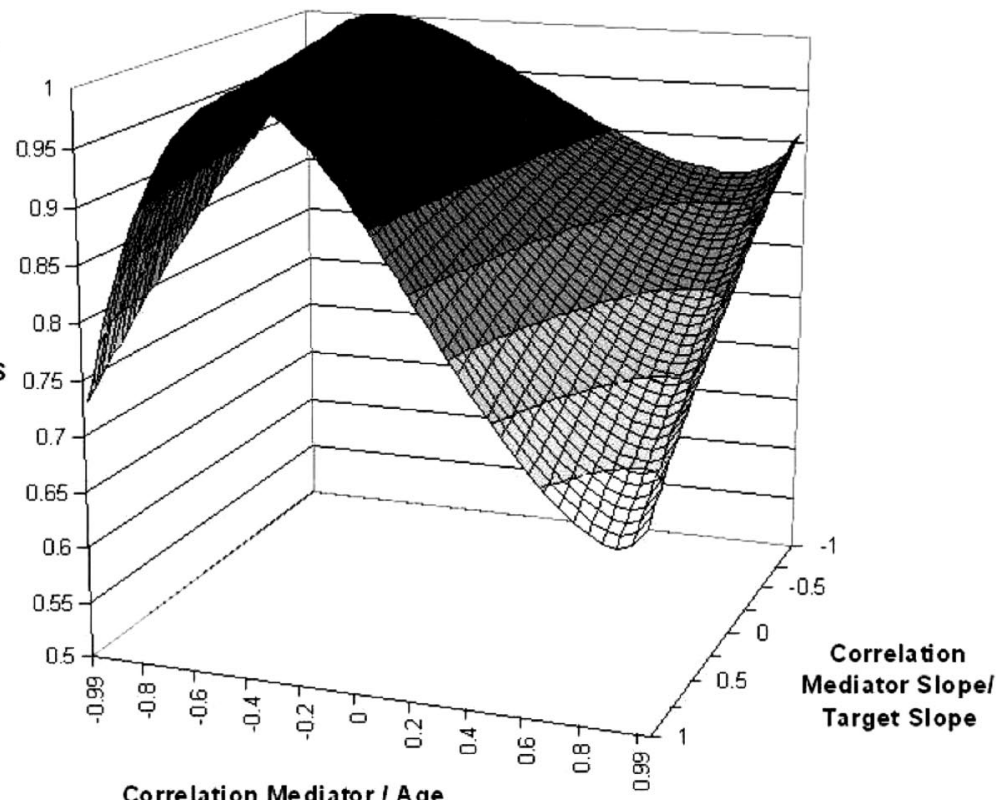

$\mathrm{B}$

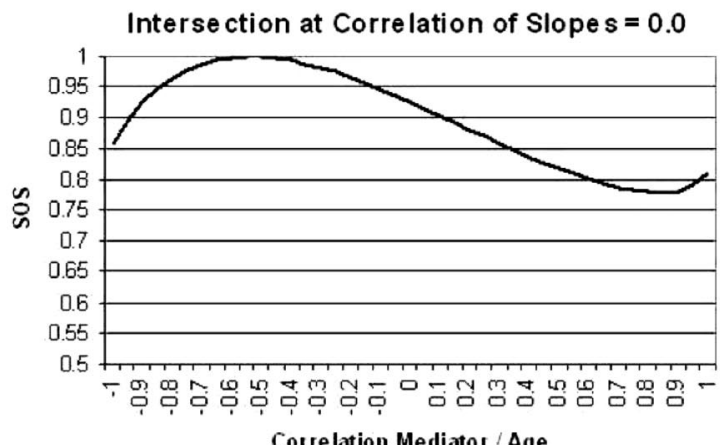

C

Intersection at SOS $=1.0$

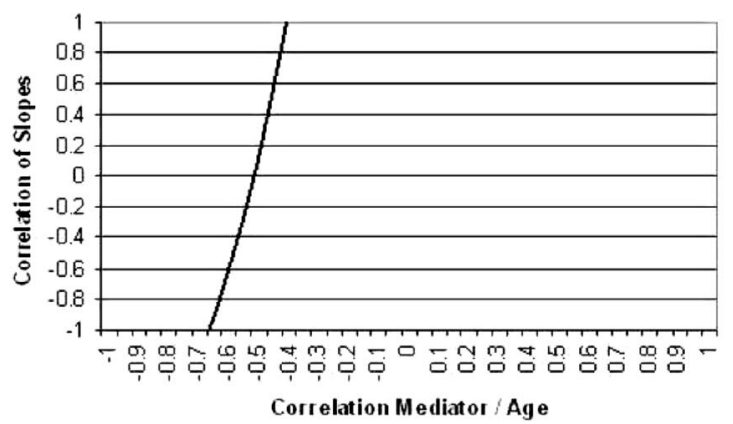

Figure 2. Shared over simple effects (SOS) as a function of slope-slope and age-mediator correlations Variances of level are set to 100, variances of slopes are set to 50, error variance for both variables is set to 25 , and the correlation of the target variable $Y$ with time is set to -0.25 , corresponding to a mean change of roughly $-10 \mathrm{~T}$-score units in 20 years. The mean change of the mediator is adapted to express the mediator-age correlation. (A) SOS as a function of the longitudinal slope-slope and the cross-sectional age-mediator correlations. (B) The slice from Panel A where the slope-slope correlation is 0. Although the two change processes are not linked, SOS take on high values and still depend on the age-mediator correlation. (C) The slice from Panel A when SOS are 1.0 (i.e., complete mediation according to cross-sectional age variance extraction). Complete mediation can occur with slope-slope correlations ranging from -1 to 1 . 
perceptual speed). To reiterate, in this empirically realistic situation, CAVE would suggest maximum interdependence between the two time-based processes, despite the fact that individual differences in change are uncorrelated.

Likewise, we can take a different slice of Panel A by inspecting slope-slope correlations and age-mediator correlations when SOS equal unity (see Panel $\mathrm{C}$ ). We note that for any slope-slope correlation in the complete range from -1.0 to 1.0 , there is a corresponding mediator-age correlation in the relatively small range from -0.65 to -0.48 that yields complete mediation according to CAVE. Hence, with SOS at maximum, slope-slope correlations can take on any value from -1 to 1 .

\section{Example 2: Sensitivity of SOS to Latent Growth Curve Parameters}

So far, we have shown that SOS are a function of additional LGCM parameters beyond the correlated changes $\operatorname{cov} S_{Y}, S_{X}$, and that these additional parameters can indeed dominate SOS. Nevertheless, under some conditions, SOS may contain information about the slope-slope correlations. One such case is represented by the following parameter settings: The variances of the intercepts are set to 100 and the variances of the slopes are set to 200, twice the intercept variance. Error variance is again set to 25 , and the intercept-to-slope correlations are all set to zero. The mean changes of the mediator $X$ and the target $Y$ are set to -14 and -5 , respectively. The covariance of intercepts is set to -3 . Note that a parameter constellation in which slope variances are twice as large as intercept variances is unlikely to represent real longitudinal data in adult cognitive development in an observational setting, which were the target of the first example.

Panel A of Figure 3 displays SOS for slope-slope correlations ranging from 0 to 1 under these assumptions. Every possible positive value of correlated changes corresponds to exactly one value of SOS, and the curve increases monotonically. Thus, in this situation, SOS show the desired relation to correlated changes. However, when we change the parameter settings slightly, the situation is drastically altered. The parameters of Panel B in Figure 3 are identical to those of Panel A, except that the mean change for the target variable $Y$ is -5 instead of -2.5 . Note that this alteration corresponds to only a quarter of the variable's standard deviation at the first measurement occasion and only $17.7 \%$ of the variable's standard deviation for the slope. Never-

Figure 3. Sensitivity of shared over simple effects (SOS) to slight changes in parameter constellations. Panel A shows SOS for slope-slope correlations ranging from 0 to 1 . The variances of the intercepts are 100 , the covariance of the intercepts is -3 , the variances of the slopes are 200, the error variance is 25 , and all the intercept-slope correlations are 0 . The mean change of the target variable $X$ is -14 , and the mean change of the mediator variable $Y$ is -5 . Under these conditions, the relationship between SOS and slope-slope correlations is monotonic and positive, as mandated by theory. However, Panels B and C illustrate that this functional form is altered by slight changes in parameters. In Panel B, the mean change of the target variable $X$ is reduced from -5 to -2.5 (1/4 of the standard deviation, leaving everything else constant). In Panel C, the level covariance is increased from -3 to 60 (correlation from close to 0 to 0.6 , leaving everything else constant).
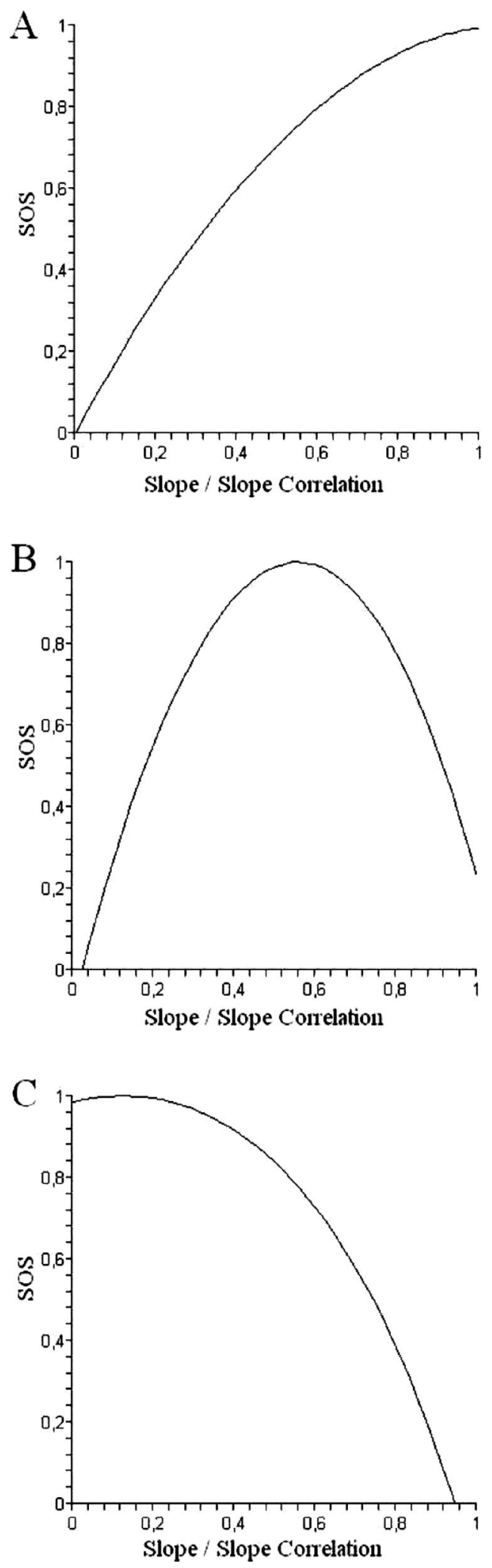
theless, this change substantially modifies the relation between SOS and slope-slope correlations: Although the increase of SOS is steeper for low slope-slope correlations, it reaches a peak at a slope-slope correlation of 0.55 and decreases thereafter. Any given value of SOS between 0.2 and 1 thus corresponds to two totally different slope-slope correlations; for instance, a value of $\mathrm{SOS}=0.4$ may correspond either to a moderate correlation of .18 or to a very high correlation of .95 . With a marginal shift in the mean change of the target variable $Y$, the relation between SOS and correlated change has become extremely ambiguous.

Finally, Panel $\mathrm{C}$ illustrates a further change in parameter values resulting in a situation in which SOS prevailingly decrease as slope-slope correlations increase. This time, the mean changes of $X$ and $Y$ are the same as in Panel A (viz., -14 and -5 ), but the level-level covariance has been set to 60 , which corresponds to a correlation of .6. All other parameters are identical to those of Panel A; in particular, the growth processes within either variable have remained unchanged. Now, SOS are informative about correlated change, but the other way around: Higher values for SOS correspond to lower slope-slope correlations. In this situation, CAVE yields paradoxical results: Shared effects approximate simple effects as slope-slope correlations are reduced from positive values to zero.

\section{Discussion}

Extending the work by Kalveram (1965), Hertzog (1985), and Hofer et al. (2006), we formally scrutinized the common practice of CAVE from the perspective of correlated linear changes in a bivariate LGCM. Our analysis shows that a candidate mediator variable can fully mediate cross-sectional age differences in CAVE, yet not manifest any correlated longitudinal change with the target variable. Instead, the influence of slope-slope correlations on SOS is easily offset by mean changes or by the correlation between the mediator and the target variable at baseline. Next, we explored whether SOS are positively related to slope-slope correlations under empirically realistic conditions. Using parameter values from longitudinal research on adult cognitive development, we found that complete mediation of age-related variance in the target variable (i.e., $\mathrm{SOS}=1.0$ ) could occur for any possible value of the slope-slope correlation (see Figure 2, Panel C). Also, when slope-slope correlations were fixed to zero, age mediation continued to be substantial (see Figure 2, Panel B).

Finally, we searched for particular parameter constellations where the slope-slope correlation does indeed exert a monotonically positive effect on SOS and presented the results for one such constellation (Figure 3, Panel A). However, when exploring the parameter space around this constellation, we noted that slight changes in just one of the other parameters transformed the function relating the slope-slope correlation to SOS from monotonically increasing (Panel A) to curvilinear (Panel B) or virtually monotonically decreasing (Panel C). Thus, the relation between SOS and the slope-slope correlation was found to be highly sensitive to minor parameter changes.

Our results are consistent with earlier explorations of the relation between longitudinal age changes and cross-sectional age differences (e.g., Baltes \& Labouvie, 1973; Cole \& Maxwell, 2003; Hertzog, 1985; Hofer et al., 2006; Maxwell \& Cole, 2007; cf. MacKinnon \& Fairchild, 2009). In line with Maxwell and Cole
(2007), who showed that cross-sectional estimates of longitudinal mediation are severely biased under a large variety of conditions, we conclude that CAVE is not a suitable research tool for inferring either (a) that developmental trends in variables are similar between variables or (b) that development in a mediator variable determines development in an outcome variable. Instead, CAVE can produce results that, on one hand, indicate mediation and, on the other hand, indicate a lack of mediation for reasons having nothing to do with whether developmental changes in two variables are correlated. Although parameter constellations resulting in a monotonically increasing relation between the slope-slope correlation and SOS exist, such local constellations (see Figure 3) are based on overly strict and unrealistic assumptions. Furthermore, researchers generally do not know what region of the SOS space is occupied by a given data set. The indeterminacy of results when using CAVE argues against its use.

This conclusion has profound consequences for the existing literature in the psychology of aging. One cannot conclude that longitudinal changes in the mediator variable and the target variable are correlated whenever CAVE shows that the mediator variable predicts a substantial amount of cross-sectional agerelated variance in the target variable. As a descriptive statement, it may be informative to report that some percentage of crosssectional age-related variance in one form of behavior is reduced by statistical control of another form of behavior. However, such a statement is typically interpreted as supporting or even demonstrating developmental causation; that is, as demonstrating that the mediator variable acts as the proximal cause of age-related changes in the target variable. Accurate descriptive statements based on CAVE such as, "[f] or reasoning, $78.6 \%$ of the agerelated variance was associated with speed" (Verhaeghen \& Salthouse, 1997, p. 241), do not permit the conclusion that age-related changes in the mediator variable (e.g., perceptual speed) determine changes in the outcome variable (e.g., reasoning). Furthermore, greater correlated change does not necessarily translate into greater SOS when CAVE is compared for multiple mediators. Rather, the variable with the strongest age correlation is likely to end up being perceived as the key mediator, even if its sensitivity to age is causally unrelated to the age-related changes under study (see also Hofer et al., 2006; Lindenberger \& Pötter, 1998).

Our findings are relevant to the fact that empirical studies of cognitive aging have found inconsistencies between longitudinal and cross-sectional results. For example, perceptual speed appears to be a stronger mediator of age effects on memory and fluid intelligence in cross-sectional data than in longitudinal data (e.g., Hertzog, Dixon, Hultsch, \& MacDonald, 2003; Hultsch et al., 1998; Lempke \& Zimprich, 2005; Lindenberger \& Ghisletta, 2009; Lindenberger, Mayr, \& Kliegl, 1993; Zimprich \& Martin, 2002). Likewise, measures of sensory functioning almost fully mediate chronological age effects in intelligence in cross-sectional data (Baltes \& Lindenberger, 1997; Lindenberger \& Baltes, 1994) but show only moderately correlated change in longitudinal data (Anstey, Hofer, \& Luszcz, 2003; Ghisletta \& Lindenberger, 2005; Lindenberger \& Ghisletta, 2009). On the basis of Equation 2, it is reasonable to assume that the strong mean age trends in perceptual speed and sensory functions contribute to these cross-sectional mediation effects (i.e., SOS). When interindividual differences in 
intraindividual change are analyzed directly, this mean-induced component of cross-sectional covariation is removed. ${ }^{3}$

Given its brittle and volatile link to correlated change, CAVE is more of a hindrance than a help in the quest to delineate the temporal ordering and causal structure of behavioral change (see also Maxwell \& Cole, 2007). We discourage the use of CAVE for this purpose. Proportions of "explained age-related variance" obtained with CAVE have tended to create confusion among developmental researchers, especially if these proportions turned out to be impressively large. To reiterate, when $78.6 \%, 80 \%$, or even $100 \%$ of the age-related variance in reasoning is associated with perceptual speed according to CAVE, this result does not imply that (a) changes in speed are correlated with changes in reasoning, (b) changes in speed precede changes in reasoning, or (c) changes in speed cause changes in reasoning. Conversely, finding a low value for SOS in CAVE does not imply that (a) changes in speed are uncorrelated with changes in reasoning, (b) changes in speed do not precede changes in reasoning, or (c) changes in speed are functionally unrelated to changes in reasoning.

\section{Limitations and Assumptions of the Present Analysis}

Our formal analysis of CAVE is based on several assumptions. First, we assume that the two change processes are linear and that variables are drawn from a multivariate normal distribution. The LGCM we adopted for formal analysis imposes further restrictions on the structure of change; namely, that errors are uncorrelated and that the growth process governs both first- and second-order moments. Second, and in a related fashion, the present analysis assumes sample homogeneity in the sense that the LGCM provides an accurate description of the change trajectories of all individuals in the population and that slope-slope correlations based on interindividual differences in change represent valid approximations to interdependencies in change within each of the individuals in the sample under study.

We note, however, that the assumptions of linearity, multivariate normality, and sample homogeneity coincide fully with the assumptions of all the statistical procedures commonly used to implement CAVE, be it hierarchical linear regression, linear structural equation modeling of mediation, path analysis, or commonality analyses (e.g., Pedhazur, 1982). That is, the assumptions of our formal critique of the method are not stronger than the assumptions inherent to the method itself.

\section{Measuring What Matters in Developmental Research}

From a more general methodological perspective, interpreting cross-sectional age differences in terms of age-related changes rests on the assumption that period and cohort effects are absent, that sampling bias does not covary with age, and that individual development can be captured by population parameters. All of these assumptions are likely to be wrong. Cross-sectional analyses provide a convenient basis for inferring whether a variable's central tendency depends on age, cohort, or both (Hertzog, 1996). However, when it comes to understanding multivariate behavioral change, there is no effective substitute for change-oriented designs and statistical analyses, time consuming and resource demanding as they may be. As researchers with an interest in the antecedents and consequences of behavioral change, we should measure this change at the individual level as precisely and adequately as we can. In this context, research on statistical power has begun to provide versatile tools for optimizing the efficiency of multivariate longitudinal designs (Hertzog et al., 2006; Hertzog, Lindenberger, Ghisletta, \& von Oertzen, 2008; von Oertzen, 2010).

As noted by Nesselroade (1991) and Molenaar (2009), developmental research for far too long has adopted a surrogate approach to the study of individual behavior and development, with between-person differences standing in for within-person changes and for between-person differences in within-person change (see also Baltes \& Labouvie, 1973; Borsboom, Mellenbergh, \& van Heerden, 2003; Lautrey, 2003; Lindenberger \& von Oertzen, 2006; Molenaar, Huizenga, \& Nesselroade, 2003). It is generally not known whether multivariate structures based on between-person differences are valid approximations to the structure of change within a given individual. People may differ in the constellation of causal factors contributing to their behavioral change, and the degree of congruence between within-person structures and between-person structures may vary by age (e.g., Brose, Schmiedek, Lövdén, Molenaar, \& Lindenberger, 2010). In-depth assessments of individual change trajectories are needed to shed light on these unresolved issues. Painful as it may be, given the temporal efficiency of the cross-sectional design, CAVE cannot be regarded as a useful first step on this path.

\footnotetext{
${ }^{3}$ In the field of child development, analogous discrepancies appear to exist. For instance, on the basis of cross-sectional analyses, Fry and Hale (1996) suggested that almost half of the age-related increase in fluid intelligence from childhood to early adulthood is mediated by individual differences in processing speed and working memory. Again, analyses of interindividual differences in intraindividual change based on longitudinal data suggest that the developmental association is weaker than extrapolated on the basis of cross-sectional data (e.g., Schneider, Schumann-Hengsteler, \& Sodian, 2005).
}

\section{References}

Anstey, K. J., Hofer, S. M., \& Luszcz, M. A. (2003). A latent growth curve analysis of late-life sensory and cognitive over 8 years: Evidence for specific and common factors underlying change. Psychology and Aging, 18, 714-726. doi: 10.1037/0882-7974.18.4.714

Baltes, P. B., \& Labouvie, G. V. (1973). Adult development of intellectual performance: Description, explanation, modification. In C. Eisdorfer \& M. Lawton (Eds.), The psychology of adult development and aging (pp. 157-219). Washington, DC: American Psychological Association. doi: 10.1037/10044-009

Baltes, P. B., \& Lindenberger, U. (1997). Emergence of a powerful connection between sensory and cognitive functions across the adult life span: A new window into the study of cognitive aging? Psychology and Aging, 12, 12-21. doi: 10.1037/0882-7974.12.1.12

Baltes, P. B., Reese, H. W., \& Nesselroade, J. R. (1988). Life-span developmental psychology: Introduction to research methods. Hillsdale, NJ: Erlbaum. (Original work published 1977)

Borsboom, D., Mellenbergh, G. J., \& van Heerden, J. (2003). The theoretical status of latent variables. Psychological Review, 110, 203-219. doi: 10.1037/0033-295X.110.2.203

Brose, A., Schmiedek, F., Lövdén, M., Molenaar, P. C. M., \& Lindenberger, U. (2010). Adult age differences in covariation of motivation and working memory performance: Contrasting between-person and withinperson findings. Research in Human Development, 7, 61-78. doi: $10.1080 / 15427600903578177$ 
Cole, D. A., \& Maxwell, S. E. (2003). Testing mediational models with longitudinal data: Questions and tips in the use of structural equation modeling. Journal of Abnormal Psychology, 112, 558-577. doi: 10.1037/0021-843X.112.4.558

Collins, L. M., Graham, J. W., \& Flaherty, B. P. (1998). An alternative framework for defining mediation. Multivariate Behavioral Research, 33, 295-312. doi: 10.1207/s15327906mbr3302_5

Craik, F. I. M., \& Bialystok, E. E. (Eds.). (2008). Lifespan cognition: Mechanisms of change. Oxford, United Kingdom: Oxford University Press.

de Frias, C. M., Lövdén, M., Lindenberger, U., \& Nilsson, L.-G. (2007). Revisiting the dedifferentiation hypothesis with longitudinal multicohort data. Intelligence, 35, 381-392. doi: 10.1016/j.intell.2006.07.011

Fairchild, A. J., MacKinnon, D. P., Taborga, M. P., \& Taylor, A. B. (2009). $R^{2}$ effect-size measures for mediation analysis. Behavior Research Methods, 41, 486-498. doi: 10.3758/BRM.41.2.486

Flavell, J. H. (1992). Cognitive development: Past, present, and future. Developmental Psychology, 28, 998-1005. doi: 10.1037/00121649.28.6.998

Fry, A. F., \& Hale, S. (1996). Processing speed, working memory, and fluid intelligence: Evidence for a developmental cascade. Psychological Science, 7, 237-241. doi: 10.1111/j.1467-9280.1996.tb00366.x

Ghisletta, P., \& Lindenberger, U. (2005). Exploring structural dynamics within and between sensory and intellectual functioning in old and very old age: Longitudinal evidence from the Berlin Aging Study. Intelligence, 33, 555-587. doi: 10.1016/j.intell.2005.07.002

Gollob, H. F., \& Reichardt, C. S. (1987). Taking into account of time lags in causal models. Child Development, 58, 80-92. doi: 10.2307/1130293

Hertzog, C. (1985). An individual differences perspective: Implications for cognitive research in gerontology. Research on Aging, 7, 7-45. doi: $10.1177 / 0164027585007001002$

Hertzog, C. (1996). Research design in studies of aging and cognition. In J. E. Birren \& K. W. Schaie (Eds.), Handbook of the psychology of aging (pp. 24-37). San Diego, CA: Academic Press.

Hertzog, C., Dixon, R. A., Hultsch, D. F., \& MacDonald, S. W. S. (2003). Latent change models of adult cognition: Are changes in processing speed and working memory associated with changes in episodic memory? Psychology and Aging, 18, 755-769. doi: 10.1037/08827974.18.4.755

Hertzog, C., Lindenberger, U., Ghisletta, P., \& Oertzen, T. von (2006). On the power of multivariate latent growth curve models to detect correlated change. Psychological Methods, 11, 244-252. doi: 10.1037/1082989X.11.3.244

Hertzog, C., Lindenberger, U., Ghisletta, P., \& Oertzen, T. von (2008). Evaluating the power of latent growth curve models to detect individual differences in change. Structural Equation Modeling, 15, 541-563. doi: 10.1080/10705510802338983

Hofer, S. M., Flaherty, B. P., \& Hoffmann, L. (2006). Cross-sectional analysis of time-dependent data: Mean-induced association in ageheterogeneous samples and an alternative method based on sequential narrow age-cohort samples. Multivariate Behavioral Research, 41, 165187. doi: $10.1207 / \mathrm{s} 15327906 \mathrm{mbr} 4102 \_4$

Hofer, S. M., \& Sliwinski, M. J. (2001). Understanding ageing: An evaluation of research designs for assessing the interdependence of ageingrelated changes. Gerontology, 47, 341-352. doi: 10.1159/000052825

Hultsch, D. F., Hertzog, C., Dixon, R. A., \& Small, B. J. (1998). Memory change in the aged. Cambridge, United Kingdom: Cambridge University Press.

Kagan, J. (1980). Perspectives on continuity. In J. O. G. Brim \& J. Kagan (Eds.), Constancy and change in human development (pp. 26-74). Cambridge, MA: Harvard University Press.

Kalveram, K. T. (1965). Die Veränderung von Faktorenstrukturen durch "simultane Überlagerung" [The modification of factor structures by simultaneous superimposition]. Archiv für die gesamte Psychologie, 17, 296-305.

Lautrey, J. (2003). A pluralistic approach to cognitive differentiation and development. In R. J. Sternberg, J. Lautrey, \& T. I. Lubart (Eds.), Models of intelligence: International perspectives (pp. 117-131). Washington, DC: American Psychological Association.

Lempke, U., \& Zimprich, D. (2005). Longitudinal changes in memory performance and processing speed in old age. Aging, Neuropsychology, and Cognition, 12, 57-77.

Lindenberger, U., \& Baltes, P. B. (1994). Sensory functioning and intelligence in old age: A strong connection. Psychology and Aging, 9, 339-355. doi: 10.1037/0882-7974.9.3.339

Lindenberger, U., \& Ghisletta, P. (2009). Cognitive and sensory declines in old age: Gauging the evidence for a common cause. Psychology and Aging, 24, 1-16. doi: 10.1037/a0014986

Lindenberger, U., Mayr, U., \& Kliegl, R. (1993). Speed and intelligence in old age. Psychology and Aging, 8, 207-220. doi: 10.1037/08827974.8.2.207

Lindenberger, U., \& Oertzen, T. von (2006). Variability in cognitive aging: From taxonomy to theory. In F. I. M. Craik \& E. Bialystok (Eds.), Lifespan cognition: Mechanisms of change (pp. 297-314). Oxford, United Kingdom: Oxford University Press.

Lindenberger, U., \& Pötter, U. (1998). The complex nature of unique and shared effects in hierarchical linear regression: Implications for developmental psychology. Psychological Methods, 3, 218-230. doi: 10.1037/1082-989X.3.2.218

MacKinnon, D. P., \& Fairchild, A. J. (2009). Current directions in mediation analysis. Current Directions in Psychological Science, 18, 16-20. doi: 10.1111/j.1467-8721.2009.01598.x

Maxwell, S. E., \& Cole, D. A. (2007). Bias in cross-sectional analyses of longitudinal mediation. Psychological Methods, 12, 23-44. doi 10.1037/1082-989X.12.1.23

McArdle, J. J., \& Nesselroade, J. R. (2003). Growth curve analyses in contemporary psychological research. In J. Schinka \& W. Velicer (Eds.), Comprehensive handbook of psychology: Research methods in psychology (Vol. 2, pp. 447-480). New York: Pergamon Press.

Molenaar, P. C. M. (2009). The new person-specific paradigm in psychology. Current Directions in Psychological Science, 18, 112-117. doi: 10.1111/j.1467-8721.2009.01619.x

Molenaar, P. C. M., Huizenga, H. M., \& Nesselroade, J. R. (2003). The relationship between the structure of inter-individual and intraindividual variability: A theoretical and empirical vindication of developmental systems theory. In U. Staudinger \& U. Lindenberger (Eds.), Understanding human development: Lifespan psychology in exchange with other disciplines (pp. 339-360). Dordrecht, the Netherlands: Kluwer Academic.

Nesselroade, J. R. (1991). The warp and the woof of the developmental fabric. In R. Downs, L. Liben, \& D. Palermo (Eds.), Visions of aesthetics, the environment and development: The legacy of Joachim Wohlwill (pp. 213-240). Hillsdale, NJ: Erlbaum.

Oertzen, T. von (2010). Power equivalence in structural equation modeling. British Journal of Mathematical and Statistical Psychology, 63, 257-272. doi: 10.1348/000711009X441021

Pedhazur, E. J. (1982). Multiple regression in behavioral research. New York: Holt.

Rabbitt, P. (1993). Does it all go together when it goes? The Nineteenth Bartlett Memorial Lecture. The Quarterly Journal of Experimental Psychology, 46, 385-434.

Salthouse, T. A. (1994). How many causes are there of aging-related decrements in cognitive functioning? Developmental Review, 14, 413437. doi: $10.1006 /$ drev.1994.1016

Salthouse, T. A. (1996). The processing-speed theory of adult age differences in cognition. Psychological Review, 103, 403-428. doi: 10.1037/ 0033-295X.103.3.403 
Schaie, K. W. (1996). Adult intellectual development: The Seattle Longitudinal Study. New York: Cambridge University Press.

Schneider, W., Schumann-Hengsteler, R., \& Sodian, B. (Eds.). (2005). Young children's cognitive development: Interrelationships among executive functioning, working memory, verbal ability, and theory of mind. Mahwah, NJ: Erlbaum.

Sliwinski, M., \& Hofer, S. (1999). How strong is the evidence for mediational hypotheses of age-related memory loss? Gerontology, 45, 351354. doi: $10.1159 / 000022120$

Verhaeghen, P., \& Salthouse, T. A. (1997). Meta-analyses of age-cognition relations in adulthood: Estimates of linear and non-linear age effects and structural models. Psychological Bulletin, 122, 231-249. doi: 10.1037/ 0033-2909.122.3.231

Wohlwill, J. F. (1970). The age variable in psychological research. Psychological Review, 77, 49-64. doi: 10.1037/h0028600

Wohlwill, J. F. (1973). The study of behavioral development. New York: Academic Press.

Zimprich, D., \& Martin, M. (2002). Can longitudinal changes in processing speed explain longitudinal age changes in fluid intelligence? Psychology and Aging, 17, 690-695. doi: 10.1037/0882-7974.17.4.690

\section{Appendix A}

\section{Expressing Cross-Sectional Variances in Terms of a Linear Growth Process}

Hofer et al. (2006) expressed the covariance between two variables in a cross-sectional data set as expectations of a bivariate latent growth curve model (LGCM; see Equation 2 in Hofer et al., 2006) and arrived at the conclusion that "associations between time-dependent processes may arise from trends in mean level even in the absence of correlated rates of change and correlated initial individual differences" (p. 171). As shown here (Remark 5), Equation 1 of the present article can be derived from Hofer et al.'s (2006) Equation 2 by distributing expectations over terms and simplifying. Doing so permits researchers to compute the cross-sectional correlation implied by any given bivariate LGCM and to analytically study the relative contributions of different LGCM components.

In this line, we express the cross-sectional variance and covariance of a mediator $X$ and a dependent variable $Y$ in terms of the parameters of a linear growth process; that is, we assume that $X$ and $Y$ are given by a linear model:

$$
\begin{aligned}
& Y(t)=I_{Y}+t \cdot S_{Y}+\operatorname{error}_{Y}(t) \\
& X(t)=I_{X}+t \cdot S_{X}+\operatorname{error}_{X}(t) .
\end{aligned}
$$

For ease of notation, we write in the following

$$
\int_{r} f(r) \omega(r):=\int_{-\infty}^{\infty} \cdots \int_{-\infty}^{\infty}(2 \pi)^{\frac{-n}{2}} e^{\frac{-x_{i}^{2}}{2}-\cdots \frac{-x_{n}^{2}}{2}} f(r) d r_{1} \cdots d r_{n}
$$

for integrals over $n$ Gaussian distribution.

Let $\mu$ be the mean vector and $M$ be the covariance matrix of the four latent variables (two levels $I_{Y}$ and $I_{X}$ and two slopes $S_{Y}$ and $S_{X}$ ) of $X$ and $Y$. Let $C$ be the Cholesky decomposition of $M$; that is,

$$
C=\left(\begin{array}{cccc}
\mathrm{c}_{1,1} & 0 & 0 & 0 \\
\mathrm{c}_{2,1} & \mathrm{c}_{2,2} & 0 & 0 \\
\mathrm{c}_{3,1} & \mathrm{c}_{3,2} & \mathrm{c}_{3,3} & 0 \\
\mathrm{c}_{4,1} & \mathrm{c}_{4,2} & \mathrm{c}_{4,3} & \mathrm{c}_{4,4}
\end{array}\right)
$$

with $C C^{T}=M$.

Let $r=\left(r_{1}, \ldots, r_{4}\right)$ be four Gaussian distributed random variables. Then, the four parameters $I_{Y}, I_{X}, S_{Y}$, and $S_{X}$ can be expressed by:

$$
\left(I_{Y}, I_{X}, S_{Y}, S_{X}\right)=C r+\mu .
$$

We assume normally distributed error terms for each measurement occasion of $Y$ and $X$, with mean zero and standard deviation $\theta_{Y}$ and $\theta_{X}$, respectively. We express these error terms by $\operatorname{error}_{j}(t)=r_{4+j}^{(t)} \theta_{j}, j=1,2$, where $r_{5}^{(t)}$ and $r_{6}^{(t)}$, respectively, are Gaussian variables that are independently distributed across time. 
Substituting $\mathrm{Cr}$ into Equation A1, we express $Y(t)$ and $X(t)$ hence as:

$$
\begin{array}{lllll}
Y(t) & =\left(\mu_{I_{Y}}+r_{1} c_{1,1}\right) & +\left(\mu_{S_{Y}}+r_{1} c_{3,1}+r_{2} c_{3,2}+r_{3} c_{3,3}\right) & t+r_{5}^{(t)} \theta_{Y} \\
X(t)=\left(\mu_{I_{X}}+r_{1} c_{2,1}+r_{2} c_{2,2}\right) & +\left(\mu_{S_{X}}+r_{1} c_{4,1}+r_{2} c_{4,2}+r_{3} c_{4,3}+r_{4} c_{4,4}\right) & t+r_{6}^{(t)} \theta_{X}
\end{array} .
$$

Let time $t$ be scaled from 0 to 1 , assuming an uniform distribution. The mean of time is $\mu_{t}=\int_{0}^{1} t d t=\frac{1}{2}$. Furthermore, $\int_{0}^{1} t^{2} d t=\frac{1}{3}$, and the variance of time is $\operatorname{var}_{t}=\int_{0}^{1}\left(t-\mu_{t}\right)^{2} d t=\frac{1}{12}$.

Lemma 1. Variances and means of $\mathrm{X}$ and $\mathrm{Y}$ are given by:

$$
\begin{aligned}
\mu_{X} & =\mu_{I_{X}}+\frac{1}{2} \mu_{S_{X}}, \\
\mu_{Y} & =\mu_{I_{Y}}+\frac{1}{2} \mu_{S_{Y}}, \\
\operatorname{var}_{X} & =\operatorname{var}_{I_{X}}+\frac{1}{3} \operatorname{var}_{S_{X}}+\operatorname{cov}_{I_{X}, S_{X}}+\frac{1}{12} \mu_{S_{X}}^{2}+\theta_{X}^{2}, \\
\operatorname{var}_{Y} & =\operatorname{var}_{I_{Y}}+\frac{1}{3} \operatorname{var}_{S_{Y}}+\operatorname{cov}_{I_{Y}, S_{Y}}+\frac{1}{12} \mu_{S_{Y}}^{2}+\theta_{Y}^{2} .
\end{aligned}
$$

Proof. The means are obvious. For the variances, we give a slightly more general proof here than usual. This proof shows the statement independently of observation density, in particular even for continuous $t$.

Integrating the term $\left(Y(t)-\mu_{Y}\right)^{2}$ over time and the Gaussian distributions yields the variance of $Y$ :

$$
\begin{aligned}
\operatorname{var}_{Y} & =\int_{0}^{1} \int_{r}\left(Y(t)-\mu_{I_{Y}}-\frac{1}{2} \mu_{S_{Y}}\right)^{2} \omega(r) d t \\
& =\int_{0}^{1} \int_{r}\left(r_{1} c_{1,1}+\left(r_{1} c_{3,1}+r_{2} c_{3,1}+r_{3} c_{3,3}\right) t+\left(t-\frac{1}{2}\right) \mu_{S_{Y}}+r_{5}^{(t)} \theta_{Y}\right)^{2} \omega(r) d t .
\end{aligned}
$$

The integral $\int_{r} r_{i} r_{j} \omega(r)$ for $i \neq j$ is zero, whereas $\int_{r} r_{i}^{2} \omega(r)=\int_{r} 1 \cdot \omega(r)=1$. Using $\int_{0}^{1} t d t=\frac{1}{2}, \int_{0}^{1} t^{2} d t=\frac{1}{3}$, and $\int_{0}^{1}\left(t-\frac{1}{2}\right)^{2} d t=\frac{1}{12}$, the expression simplifies to:

$$
\begin{aligned}
\operatorname{var}_{Y} & =\int_{0}^{1}\left(c_{1,1}^{2}+2 c_{1,1} c_{3,1} t+\left(t-\frac{1}{2}\right)^{2} \mu_{S_{Y}}^{2}+c_{3,1}^{2} t^{2}+c_{3,2}^{2} t^{2}+c_{3,3}^{2} t^{2}+\theta_{Y}^{2}\right) d t \\
& =c_{1,1}^{2}+c_{1,1} c_{3,1}+\frac{1}{12} \mu_{S_{Y}}^{2}+\frac{1}{3}\left(c_{3,1}^{2}+c_{3,2}^{2}+c_{3,3}^{2}\right)+\theta_{Y}^{2} .
\end{aligned}
$$

Since $C C^{T}=M$, we have $\operatorname{var}_{I_{Y}}=c_{1,1}^{2}, \operatorname{var}_{S_{Y}}=c_{3,1}^{2}+c_{3,2}^{2}+c_{3,3}^{2}$, and $\operatorname{cov}_{I_{Y}, S_{Y}}=c_{1,1} c_{3,1}$. We thus obtain

$$
\operatorname{var}_{Y}=\operatorname{var}_{I_{Y}}+\frac{1}{3} \operatorname{var}_{S_{Y}}+\operatorname{cov}_{I_{Y}, S_{Y}}+\frac{1}{12} \mu_{S_{Y}}^{2}+\theta_{Y}^{2}
$$

We proceed analogously for $X$. 
Lemma 2. The covariance of $\mathrm{Y}$ and $\mathrm{X}$ with time are

$$
\begin{aligned}
& \operatorname{cov}_{Y, t}=\frac{1}{12} \mu_{S_{Y}} \\
& \operatorname{cov}_{X, t}=\frac{1}{12} \mu_{S_{X}} .
\end{aligned}
$$

Proof. As in the proof of Lemma 1, we integrate the product of the two values over time and the random distributions for $Y$ :

$$
\begin{aligned}
\operatorname{cov}_{Y, t} & =\int_{0}^{1} \int_{r}\left(t-\frac{1}{2}\right)\left(Y(t)-\mu_{I_{Y}}-\frac{1}{2} \mu_{S_{Y}}\right) \omega(r) d t \\
& =\int_{0}^{1} \int_{r}\left(t-\frac{1}{2}\right)\left(r_{1} c_{1,1}+\left(r_{1} c_{3,1}+r_{2} c_{3,2}+r_{3} c_{3,3}\right) t+\left(t-\frac{1}{2}\right) \mu_{S_{Y}}+r_{5}^{(t)} \theta_{Y}\right) \omega(r) d t
\end{aligned}
$$

using the same simplifications for the integrals, we obtain

$$
\begin{aligned}
\operatorname{cov}_{Y, t} & =\int_{0}^{1}\left(t-\frac{1}{2}\right)^{2} \mu_{S_{Y}} d t \\
& =\frac{1}{12} \mu_{S_{Y} .}
\end{aligned}
$$

We proceed analogously for $X$.

Corollary 3. The correlations of $\mathrm{Y}$ and $\mathrm{X}$ with time are given by

$$
\begin{aligned}
r_{Y, t} & =\frac{\mu_{S_{Y}}}{\sqrt{12 \operatorname{var}_{I_{Y}}+4 \operatorname{var}_{S_{Y}}+12 \operatorname{cov}_{I, S_{Y}}+\mu_{S_{Y}}^{2}+12 \theta_{Y}^{2}}} \\
r_{X, t} & =\frac{\mu_{S_{X}}}{\sqrt{12 \operatorname{var}_{I_{X}}+4 \operatorname{var}_{S_{X}}+12 \operatorname{cov}_{I, S_{X}}+\mu_{S_{X}}^{2}+12 \theta_{X}^{2}}} .
\end{aligned}
$$

Solved to $\mu_{S_{Y}}$ and $\mu_{S_{X}}$, respectively, these terms can be transformed to

$$
\begin{aligned}
& \mu_{S_{Y}}=r_{Y, t} \sqrt{\frac{12 \operatorname{var}_{I_{Y}}+4 \operatorname{var}_{S_{Y}}+12 \operatorname{cov}_{I_{Y}, S_{Y}}+12 \theta_{Y}^{2}}{1-r_{Y, t}^{2}}} \\
& \mu_{S_{X}}=r_{X, t} \sqrt{\frac{12 \operatorname{var}_{I_{X}}+4 \operatorname{var}_{S_{X}}+12 \operatorname{cov}_{I_{X}, S_{X}}+12 \theta_{X}^{2}}{1-r_{X, t}^{2}}} .
\end{aligned}
$$

Proof. The correlation of $Y$ and $t$ is gained from substituting Lemma 2 into the definition of the correlation,

$$
r_{Y, t}=\frac{\operatorname{cov}_{Y, t}}{\sqrt{\operatorname{var}_{Y} \operatorname{var}_{t}}}
$$

The second statement is purely computational. 
Lemma 4. The correlation between $\mathrm{Y}$ and $\mathrm{X}$ is given by:

$$
r_{Y, X}=\frac{12 \operatorname{cov}_{I_{Y}, I_{X}}+6\left(\operatorname{cov}_{I_{Y}, S_{X}}+\operatorname{cov}_{I_{X}, S_{Y}}\right)+4 \operatorname{cov}_{S_{Y}, S_{X}}+\mu_{S_{Y}} \mu_{S_{X}}}{\sqrt{\left(12 \operatorname{var}_{I_{Y}}+4 \operatorname{var}_{S_{Y}}+12 \operatorname{cov}_{I_{Y}, S_{Y}}+\mu_{S_{Y}}^{2}+\theta_{Y}^{2}\right)\left(12 \operatorname{var}_{I_{X}}+4 \operatorname{var}_{S_{X}}+12 \operatorname{cov}_{I_{X}, S_{X}}+\mu_{S_{X}}^{2}+\theta_{X}^{2}\right)}} .
$$

Proof. As before, we compute:

$$
\begin{aligned}
\operatorname{cov}_{Y, X} & =\int_{0}^{1} \int_{r}\left(Y(t)-\mu_{I_{Y}}-\frac{1}{2} \mu_{S_{Y}}\right)\left(X(t)-\mu_{I_{X}}-\frac{1}{2} \mu_{S_{X}}\right) \omega(r) d t \\
& =\int_{0}^{1} \int_{r}\left(r_{1} c_{1,1}+\left(r_{1} c_{3,1}+r_{2} c_{3,2}+r_{3} c_{3,3}\right) t+\left(t-\frac{1}{2}\right) \mu_{S_{Y}}+r_{5}^{(t)} \theta_{Y}\right) \\
& \times\left(r_{1} c_{2,1}+r_{2} c_{2,2}+\left(r_{1} c_{4,1}+r_{2} c_{4,2}+r_{3} c_{4,3}+r_{4} c_{4,4}\right) t+\left(t-\frac{1}{2}\right) \mu_{S_{X}}+r_{6}^{(t)} \theta_{X}\right) \omega(r) d t .
\end{aligned}
$$

Eliminating the integrals yields:

$$
\begin{aligned}
\operatorname{cov}_{Y, X} & =\int_{0}^{1} c_{1,1} c_{2,1}+\left(c_{1,1} c_{4,1}+c_{3,1} c_{2,1}+c_{3,2} c_{2,2}\right) t \\
& +\left(c_{3,1} c_{4,1}+c_{3,2} c_{4,2}+c_{3,3} c_{4,3}\right) t^{2}+\left(t-\frac{1}{2}\right)^{2} \mu_{S_{Y}} \mu_{S_{X}} d t \\
& =c_{1,1} c_{2,1}+\frac{1}{2}\left(c_{1,1} c_{4,1}+c_{3,1} c_{2,1}+c_{3,2} c_{2,2}\right) \\
& +\frac{1}{3}\left(c_{3,1} c_{4,1}+c_{3,2} c_{4,2}+c_{3,3} c_{4,3}\right)+\frac{1}{12} \mu_{S_{Y}} \mu_{S_{X}} .
\end{aligned}
$$

Since $C C^{T}=M$, we have $c_{1,1} c_{2,1}=\operatorname{cov}_{I Y, I_{X}}, c_{1,1} c_{4,1}=\operatorname{cov}_{I Y, S}, c_{3,1} c_{2,1}+c_{3,2} c_{2,2}=\operatorname{cov}_{I X, S_{Y}}$, and $c_{3,1} c_{4,1}+c_{3,2} c_{4,2}+c_{3,3} c_{4,3}=\operatorname{cov}_{S_{Y}, S_{X}}$. Using these transformations, the aforementioned term simplifies to:

$$
\operatorname{cov}_{Y, X}=\operatorname{cov}_{I_{Y, I_{X}}}+\frac{1}{2}\left(\operatorname{cov}_{I_{Y}, S_{X}}+\operatorname{cov}_{I_{X}, S_{Y}}\right)+\frac{1}{3} \operatorname{cov}_{S_{Y}, S_{X}}+\frac{1}{12} \mu_{S_{Y}} \mu_{S_{X}}
$$

To compute the correlation of $Y$ and $X$, we divide this term by the square root of the variances:

$$
r_{Y, X}=\frac{\operatorname{cov}_{Y, X}}{\sqrt{\operatorname{var}_{Y} v a r_{X}}} .
$$

Remark 5. Hofer et al. (2006) gave the following equation (Equation 2 in their study):

$$
\begin{aligned}
\operatorname{Cov}(X, Y) & =E\left(L_{x i} L_{y i}+L_{x i} S_{Y} t_{i}+L_{x i} S_{y i} t_{i}+S_{X} t_{i} L_{y i}\right. \\
& +S_{X} t_{i} L_{y i}+S_{X} t_{i} S_{Y} t_{i}+S_{X} t_{i} S_{y i} t_{i}-S_{X} t_{i} S_{Y} \bar{t} \\
& +S_{x i} t_{i} L_{y i}+S_{x i} t_{i} S_{Y} t_{i}+S_{x i} t_{i} S_{y i} t_{i}-S_{x i} t_{i} S_{Y} \bar{t} \\
& \left.-S_{X} \bar{t} S_{Y} t_{i}-S_{X} \bar{t} S_{y i} t_{i}+S_{X} \bar{t} S_{Y} \bar{t}\right)
\end{aligned}
$$

In their notation, level and slope of $X$ and $Y$ are split in a constant term $\left(I_{X}, I_{Y}, S_{X}\right.$ and $S_{Y}$, without index $\left.i\right)$ and a zero-mean normally distributed term $\left(L_{x i}, L_{y i}, S_{x i}\right.$, and $S_{y i}$ with index $\left.i\right)$. The $t_{i}$ is the time at point $i$, conceived as a random variable with mean $\bar{t}$ of any distribution. After the expectation is distributed across terms, some simplifications can be made. As time is independent of level and slope, all terms that include exactly one 
growth parameter indexed by $i$ (Terms $2,4,6,9,11$, and 13) are zero; they can thus be omitted. As $\bar{t}$ is fixed, $E\left(\bar{t} t_{i}\right)=\bar{t}^{2}=E(\overline{t t})$, and Terms 12 and 14 cancel each other out. The remaining terms can be transformed to Equation A29.

\section{Appendix B}

\section{Controlling for the Mediator and SOS Effects}

Again, let $X$ be a mediator variable and $Y$ be a dependent variable following a latent growth curve model (LGCM) as given in Equation A1. In Appendix B, we compute the SOS of these two variables in terms of the LGCM.

Theorem 6. The SOS of Y mediated by X are

$$
S O S=1-\frac{12 \operatorname{var}_{Y}\left(\mu_{S_{Y}} \operatorname{var}_{X}-\mu_{S_{X}} \operatorname{cov}_{Y, X}\right)^{2}}{\left(\operatorname{var}_{Y} \operatorname{var}_{X}-\operatorname{cov}_{Y, X}^{2}\right)\left(12 \operatorname{var}_{X}-\mu_{S_{X}}^{2}\right) \mu_{S_{Y}}^{2}}
$$

with $\operatorname{var}_{\mathrm{Y}}, \operatorname{var}_{\mathrm{X}}$, and $\operatorname{cov}_{\mathrm{Y}, \mathrm{X}}$ from Lemmas 1 and 2 .

Proof. We first express the partial correlation of $Y$ and time controlling for $X$ in terms of the latent growth process:

$$
r_{Y, t \cdot X}=\frac{r_{Y, t}-r_{Y, X} r_{X, t}}{\sqrt{\left(1-r_{Y, X}^{2}\right)\left(1-r_{X, t}^{2}\right)}}
$$

The square of this partial correlation corresponds to the unique effect of age on $Y$ (Lindenberger \& Pötter, 1998; see Figure 1). The difference between the simple effect (i.e. $r_{Y, t}^{2}$ ) and the unique effect is defined as the shared effect of age and $X$ on $Y$. Shared over simple effects, or SOS, are thus:

$$
\begin{aligned}
\text { SOS } & =\frac{\text { shared }}{\text { simple }} \\
& =\frac{\text { simple }- \text { unique }}{\text { simple }} \\
& =1-\frac{\text { unique }}{\text { simple }} \\
& =1-\frac{r_{Y, t X X}^{2}}{r_{Y, t}^{2}}
\end{aligned}
$$

Simplifying this term and expressing it in terms of variances and covariances yields

$$
\begin{aligned}
S O S & =1-\frac{r_{Y, t \cdot X}^{2}}{r_{Y, t}^{2}} \\
& =1-\frac{\left(r_{Y, t}-r_{Y, X} r_{X, t}\right)^{2}}{r_{Y, t}^{2}\left(1-r_{Y, X}^{2}\right)\left(1-r_{X, t}^{2}\right)} \\
& =1-\frac{\left(1-\frac{r_{Y, X} r_{X, t}}{r_{Y, t}}\right)^{2}}{\left(1-r_{Y, X}^{2}\right)\left(1-r_{X, t}^{2}\right)}
\end{aligned}
$$




$$
\begin{gathered}
=1-\frac{\frac{\left(\operatorname{cov}_{Y, t} \operatorname{var}_{X}-\operatorname{cov}_{Y, X} \operatorname{cov}_{X, t}\right)^{2}}{\operatorname{var}_{X}^{2} \operatorname{var}_{Y} \operatorname{var}_{t}}}{\frac{\left(\operatorname{var}_{Y} \operatorname{var}_{X}-\operatorname{cov}_{Y, X}^{2}\right)\left(\operatorname{var}_{X} \operatorname{var}_{t}-\operatorname{cov}_{X, t}^{2}\right) \operatorname{cov}_{Y, t}^{2}}{\operatorname{var}_{Y}^{2} \operatorname{var}_{X}^{2} \operatorname{var}_{t}^{2}}} \\
=1-\frac{\operatorname{var}_{Y} \operatorname{var}_{t}\left(\operatorname{cov}_{Y, t} \operatorname{var}_{X}-\operatorname{cov}_{Y, X} \operatorname{cov}_{X, t}\right)^{2}}{\left(\operatorname{var}_{Y} \operatorname{var}_{X}-\operatorname{cov}_{Y, X}^{2}\right)\left(\operatorname{var}_{X} \operatorname{var}_{t}-\operatorname{cov}_{X, t}^{2}\right) \operatorname{cov}_{Y, t}^{2}} .
\end{gathered}
$$

Substituting variance of time and the covariances between $Y$ and $X$ with time, respectively, yields the statement.

For some demonstrations, we want to describe all situations where $\mathrm{SOS}=1$. The following corollary can be derived from Theorem 6:

Corollary 7. If $\operatorname{SOS}=1$, then

$$
\operatorname{cov}_{S_{Y}, S_{X}}=\frac{\mu_{S_{Y}}}{\mu_{S_{X}}}\left(3 \operatorname{var}_{I_{X}}+\operatorname{var}_{S_{X}}+3 \operatorname{cov}_{I_{X}, S_{X}}+3 \theta_{X}^{2}\right)-3 \operatorname{cov}_{I_{Y}, I_{X}}-\frac{3}{2}\left(\operatorname{cov}_{I_{Y}, S_{X}}+\operatorname{cov}_{I X, S_{Y}}\right) .
$$

Proof. Assume SOS $=1$. Then, Equation B1 is transformed to

$$
\begin{aligned}
0 & =\frac{12 \operatorname{var}_{Y}\left(\mu_{S_{Y}} \operatorname{var}_{X}-\mu_{S_{X}} \operatorname{cov}_{Y, X}\right)^{2}}{\left(\operatorname{var}_{Y} \operatorname{var}_{X}-\operatorname{cov}_{Y, X}^{2}\right)\left(12 \operatorname{var}_{X}-\mu_{S_{X}}^{2}\right) \mu_{S_{Y}}^{2}} \\
\Leftrightarrow \operatorname{cov}_{Y, X} & =\frac{\mu_{S_{Y}}}{\mu_{S_{X}}} \operatorname{var}_{X} .
\end{aligned}
$$

Substituting Equations A29 and A8 into this equation and solving for $\operatorname{cov}_{\mathrm{S}_{\mathrm{Y}}, \mathrm{S}_{\mathrm{X}}}$ yields:

$$
\begin{aligned}
\operatorname{cov}_{S_{Y}, S_{X}} & =3 \frac{\mu_{S_{Y}}}{\mu_{S_{X}}}\left(\operatorname{var}_{I_{X}}+\frac{1}{3} \operatorname{var}_{S_{X}}+\operatorname{cov}_{I_{X}, S_{X}}+\frac{1}{12} \mu_{S_{X}}^{2}+\theta_{X}^{2}\right)-3 \operatorname{cov}_{I_{Y}, I_{X}}-\frac{3}{2}\left(\operatorname{cov}_{I_{Y}, S_{X}}+\operatorname{cov}_{I_{X}, S_{Y}}\right)-\frac{3}{12} \mu_{S_{Y}} \mu_{S_{X}} \\
& =\frac{\mu_{S_{Y}}}{\mu_{S_{X}}}\left(3 \operatorname{var}_{I_{X}}+\operatorname{var}_{S_{X}}+3 \operatorname{cov}_{I_{X}, S_{X}}+3 \theta_{X}^{2}\right)-3 \operatorname{cov}_{I_{Y, I X}}-\frac{3}{2}\left(\operatorname{cov}_{I_{Y}, S_{X}}+\operatorname{cov}_{I_{X}, S_{Y}}\right)
\end{aligned}
$$

If needed, Equations A22 and A23 can be used to replace the mean slopes by the correlation of $Y$ and $X$ with time, respectively.

Received July 15, 2009

Revision received May 20, 2010

Accepted June 1, 2010 